\title{
A colour-based image segmentation method for the measurement of masticatory performance in older adults
}

\author{
Lorenzo Scalise ${ }^{1}$, Rachele Napolitano ${ }^{1}$, Lorenzo Verdenelli ${ }^{1}$, Susanna Spinsante ${ }^{2}$, Giorgio Rappelli ${ }^{3}$ \\ ${ }^{1}$ Department of Industrial Engineering and Mathematical Sciences (DIISM), Università Politecnica delle Marche, Ancona, Italy \\ 2 Department of Information Engineering (DII), Università Politecnica delle Marche, Ancona, Italy \\ ${ }^{3}$ Department of Odontostomatologic and Specialized Clinical Sciences (DISCO), Università Politecnica delle Marche, Ancona, Italy
}

\begin{abstract}
Masticatory efficiency in older adults is an important parameter for the assessment of their oral health and quality of life. This study presents a measurement method based on the automatic segmentation of two-coloured chewing gum based on a $K$-means clustering algorithm. The solution proposed aims to quantify the mixed areas of colour in order to evaluate masticatory performance in different dental conditions. The samples were provided by 'two-colour mixing' tests, currently the most used technique for the evaluation of masticatory efficacy, because of its simplicity, low acquisition times and reduced cost. The image analysis results demonstrated a high discriminative power, providing results in an automatic manner and reducing errors caused by manual segmentation. This approach thus provides a feasible and robust solution for the segmentation of chewed samples. Validation was carried out by means of a reference software, demonstrating a good correlation $\left(R^{2}=0.64\right)$ and the higher sensitivity of the proposed method $(+75 \%)$. Tests on patients with different oral conditions demonstrated that the $K$-means segmentation method enabled the automatic classification of patients with different masticatory conditions, providing results in a shorter time period (20 chewing cycles instead of 50).
\end{abstract}

\section{Section: RESEARCH PAPER}

Keywords: Measurement of masticatory efficiency; chewing gum; colour image segmentation; K-means clustering

Citation: Lorenzo Scalise, Rachele Napolitano, Lorenzo Verdenelli, Susanna Spinsante, Giorgio Rappelli, A colour-based image segmentation method for the measurement of masticatory performance in older adults, Acta IMEKO, vol. 10, no. 2, article 26, June 2021, identifier: IMEKO-ACTA-10 (2021)-02-26

Section Editor: Yasuharu Koike, Tokyo Institute of Technology, Japan

Received July 28, 2018; In final form June 9, 2021; Published June 2021

Copyright: This is an open-access article distributed under the terms of the Creative Commons Attribution 3.0 License, which permits unrestricted use, distribution, and reproduction in any medium, provided the original author and source are credited.

Corresponding author: Lorenzo Scalise, e-mail: I.scalise@pm.univpm.it

\section{INTRODUCTION}

In the twenty-first century, the population aged 65 and over has increased, and forecasts confirm this to be a continuing trend. This is mainly due to a general increase in mean life expectancy and to a decrease in the birth rate (particularly in Western countries) [1]. In addition, considerable socio-economic development over the last 50 years has contributed to a significant increase in the well-being of the population and the level of public health. Quality of life depends largely on the prevention and control of disease, and one health condition that affects the quality of life is oral health. Indeed, according to the World Health Organization [1], having a good state of oral health is about much more than just having healthy teeth; poor oral health is one of the most frequent causes of malnutrition, which can have a large impact on daily life. In particular, many activities can become difficult for older adults, such as eating adequately and satisfactorily, speaking fluently and correctly, smiling, laughing, showing one's teeth without embarrassment and maintaining gratifying family and social relationships [2].

In a recent study [3], a questionnaire survey was conducted in order to provide a global overview of oral health conditions in older people. This survey indicated that incidence of oral disease is high in low-income countries, and the use of oral health services, widely used in developed countries, is low among the older population. In addition to socio-economic status and the regularity of dental visits, it is observed that dental caries and periodontal disease constitute a considerable public health problem in the majority of countries. In particular, the consequences of these oral problems that most frequently afflict the elderly are missing teeth, dry mouth and limitations in chewing ability. For these reasons, in the present study, the association between dental status and masticatory function, especially in elderly patients, was investigated in order to identify an impaired masticatory system and evaluate the appropriate clinical treatment [4]. The results indicated a direct relationship between masticatory function and the number of remaining 
teeth. Indeed, it was demonstrated that chewing difficulties increase as the number of posterior functional units decreases, when the remaining teeth are unevenly distributed or there are spaces between the teeth. In relation to the influence of prosthetic rehabilitation on masticatory function, it was observed that those with fewer teeth tended to have them replaced with a removable partial denture (RPD) with many artificial functional dental units [5]. Subjects with fewer teeth reported greater masticatory difficulty than those with RPD. However, there is no certainty that replacing lost teeth with an RPD is efficient, as some studies have demonstrated. In fact, masticatory function is not necessarily better in patients with a minimum number of occlusal units (up to 20 teeth) and RPD. The replacement of lost posterior teeth with RPD is suggested when there are less than three pairs of posterior antagonist teeth that correspond to a number of teeth less than 20.

For all these reasons, it is clear that dentists should implement all the measures at their disposal so that this category of patient can achieve and maintain good oral health. In particular, precise, repeatable and comparable measurement methods are sought for use with this class of patient.

\section{Current methodologies}

There are a variety of methodologies describing masticatory function. In particular, there is the patient factor, which depends on a variety of personal and subjective habits. A subjective assessment is based on an individual's self-perception of chewing ability and cannot provide a complete evaluation of chewing problems, oral conditions and prosthesis quality. This method is rather weak and is not a proven test. For this reason, subjective tests using questionnaires or personal interviews to determine masticatory ability are usually supplemented with scientifically objective tests to assess masticatory efficiency [6]. The combination of the two methods provides a wider evaluation of the chewing efficiency function [7], [8].

Fragmenting tests with sieve analysis or optical scanning methods are regarded as the gold standard in assessing masticatory efficiency [9]. Usually, these tests employ a standard number of peanuts or carrots as natural test foods or silicone cubes as artificial test foods; they are chewed for a determinate time or number of cycles and are then passed through sieves with different mesh diameters. The final degree of subdivisions of the chewed sample is evaluated by measuring the median particle size (D50) [10]. Despite this technique being relatively accurate, it is not very practical; in fact, the particle size of the test foods is very small, and the mobility and sensitivity of the oral structures are reduced [11]. Furthermore, the digital image processing software used in optical scanning methods is expensive and complicated to use. It is certainly not a method that can be used on an outpatient basis, as it requires high-precision laboratory instruments.

Alternative methods using two-coloured chewing gum are considered more practical for detecting changes in dental status. These techniques can provide both the degree of colour blending and the ability to form a bolus. These 'two-colour mixing' tests have a significant correlation with the sieving method, especially in patients with impaired masticatory function [12]. The advantage of using the two-colour mixing method in comparison with the sieving method lies in its simplicity of implementation, its lower acquisition times, the relatively cheap cost and the ease of recovery after chewing. Liedberg et al. [13] evaluated the degree of blending of the two colours in different chewing cycles by means of a subjectively visual test. Prinz computerised this process with an image processing approach using custom-made software [14]. In this study, ranking the different gum samples using image processing measurements was not found to be more accurate than subjective human evaluation. Schimmel et al. [15], [16] also developed customised software, ViewGum(C), to determine the degree of colour mixing. This software calculates the hue value for each pixel in the pictures of semi-automatically segmented gum wafers and displays the standard deviation between two well-separated peaks on the hue axis [15]. The variance of the hue component in the hue, intensity and saturation (HIS) colour space was considered the measurement of mixing; a low variance indicates a greater colour mixture and, therefore, represents better masticatory performance, while a higher variance indicates inadequate mixing [16]. However, this software introduces errors due to an operator-guided procedure for the segmentation of the gum images and to the difficulty in distinguishing hue variation between the gum and the background colour, especially in well-mixed gum. Indeed, extracted data are highly dependent on the accuracy of the segmentation process, which is an essential step in computer vision and automatic pattern recognition based on image analysis [17].

The aim of the present study was to propose an automatic colour-based segmentation method to separate mixed and unmixed colours of images derived from the application of a two-colour mixing test. From the different clustering methods described in the literature [18], 'K-means clustering' was adopted for its solid application history and its rather higher efficiency in image feature classification, which has also achieved very good results for unsupervised image datasets [19], [20]. As a result, this approach can provide an automatic classification of the colour areas of interest perfectly separated from background colours.

Within this context, the chewing performance of six older patients ( $>65$ years) with different dental status were evaluated. It has been shown that this segmentation method can discriminate between dental status perfectly and can provide quantitative data by means of computerised analysis using the best possible segmentation and minimising human interaction.

\section{MATERIALS AND METHODS}

\subsection{Clinical protocol}

An experimental laboratory was used for the proposed measurement method, based on two-colour mixing tests, with the participation of six patients at the Odontostomatologic Clinic of the Polytechnic University of Marche (Ancona). The study participants were elderly patients aged between 64 and 84 years (median age of 73.2 years). After providing informed consent for clinical treatment, the patients were subject to a general dental examination, in which medical and clinical data were collected. The instruments used for the clinical evaluation were a mirror, probes (curved and straight), tweezers and a periodontal probe.

The pre-test check and the following outpatient clinic test were conducted between November 2016 and March 2017. Personal data, lifestyle habits (use of tobacco, alcohol, stress, physical activity, nutrition), familial information, and physiological and pathological anamnesis were investigated. In addition, the following clinical data were evaluated and annotated in the folder:

- Missing teeth

- Root residue

- Decayed teeth

- Filled teeth 
- Mobile teeth

- $\quad$ Fixed partial dentures (FPD)

- RPDs

- Periodontal screening and recording (PSR).

A nutritional test to investigate eating habits was completed by a specialist doctor at the Department of Clinical and Specialist Dental Sciences. The test used was the Mini Nutritional Assessment (MNA $\left.{ }^{\circledR}\right)$, a well-known and largely accepted monitoring and evaluation tool designed to identify elderly patients at risk of malnutrition based on questions on their nutritional habits [21]. Before starting the MNA®, general information relating to the patient was entered in the appropriate space at the top of the form. The screening section (MNA®-SF), consisting of six questions, was then completed. If the screening score was less than 11, an evaluation to determine the malnutrition index was carried out; if the score was $>23.5$, the patient was considered to have a normal nutritional status, and no further action was necessary, while if the score was $<23.5$, the patient was referred to a dietician or a nutritionist.

\subsection{Mixing test procedure}

After the pre-test clinical protocol had been completed, the test based on the proposed measurement method was performed. Each specimen was prepared from two large sticks of bubble gum, one yellow and one pink (saliva did not affect the gum colours). Strips $20 \mathrm{~mm}$ in length were cut from both colours and manually stuck together so that the test strip presented as 20 $\times 10 \times 2 \mathrm{~mm}^{3}$ (Figure 1-a).

For all the participants, five pairs of chewing gum sticks were prepared, and five boluses for different chewing cycles were obtained (Figure 1-b) for

$$
\begin{aligned}
& \text { - } \quad 5 \text { chewing strokes } \\
& \text { - } \quad 10 \text { chewing strokes } \\
& \text { - } \quad 20 \text { chewing strokes } \\
& \text { - } \quad 30 \text { chewing strokes } \\
& \text { - } \quad 50 \text { chewing strokes. }
\end{aligned}
$$

Each bolus was gently dabbed with a paper towel in order to eliminate any possible excess saliva and then flattened to form a

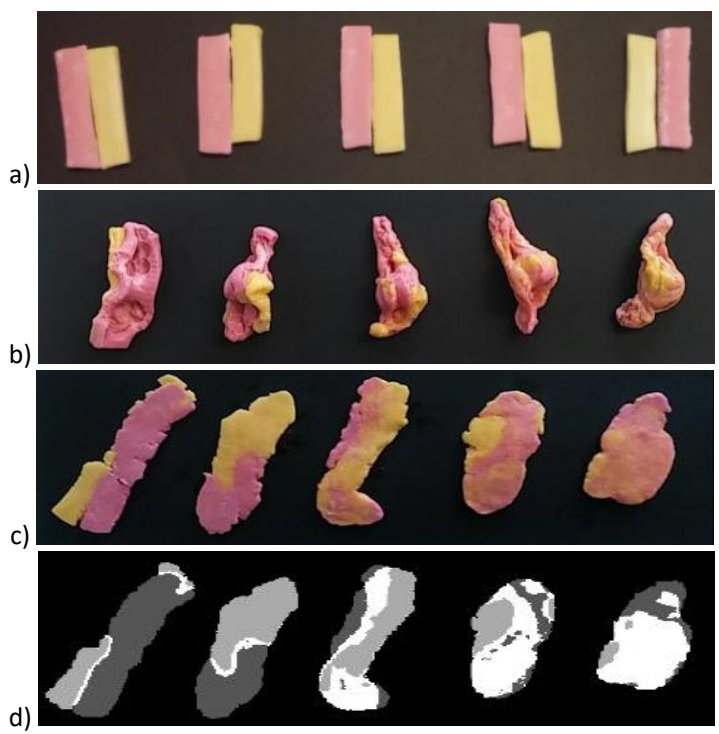

Figure 1. Five pairs of chewing gum sticks were used in this study: a) unchewed, b) bolus obtained from 5, 10, 20, 30 and 50 chewing cycles, from left to right, c) a flattened wafer and d) an image labelled by cluster index. 1-mm-thick wafer (Figure 1-c). The total duration of the test was about 5 minutes.

In the laboratory tests, an accurate digitisation of each sample was performed. The procedure was divided into two main phases:

1. An acquisition phase, in which the images of different chewed wafers were captured by means of a CCD camera (1,920 x 1,080 pixels) mounted on a smart phone (LG G2 D802). Each wafer was placed on a dark background so that the different colours could be more easily separated. For all the images acquired, the camera resolution, type of illumination and camera-to-bolus distance remained fixed.

2. Processing phases, necessary to classify an image into different colour groups. A PC (Intel ${ }^{\circledR}$ Core TM i76700HQ, 2.6 GHz, 16.0 GB) with Windows 10 Home was used to process the acquired data, and MATLAB ${ }^{\circledR}$ R2017b software enabled the colour-based segmentation.

\subsection{Image segmentation method}

K-means clustering was the method proposed to process the images in order to obtain information on the colours in the mixed and unmixed areas of the chewing gum. This method separates pixels into regions according to their properties, such as colour, intensity or texture. For each region, called a cluster, it is possible to define $\mathrm{K}$ centroids, which are placed as far from each other as possible [19].

Each point belonging to a given dataset is then assigned to the nearest centroid until no points are left. When all the points have been assigned to an initial group of centroids, the positions of the new $\mathrm{K}$ centroids are then recalculated as the barycentre of the cluster. After identifying these new $K$ centroids, a new binding has to be established between the same dataset points and the nearest new centroid. As a result of this loop, it is possible to observe that the $\mathrm{K}$ centroids change their location systematically until they stop moving.

In the present study, the optimal number of $\mathrm{K}$ clusters to be adopted was determined from the value that minimises the total intra-cluster variance [20]. This is equivalent to minimising the pairwise squared deviations of points in the same cluster:

$J=\sum_{i=1}^{c} \sum_{j=1}^{c_{i}}\left\|x_{i}-v_{j}\right\|^{2}$

where $x_{i}$ is the set of data points, $v_{j}$ is the set of centres, $\left\|x_{i}-v_{j}\right\|$ is the Euclidean distance between $x_{i}$ and $v_{j}, c_{i}$ is the number of data points in $i^{\text {th }}$ cluster and $c$ is the number of cluster centres.

In the framework of this study, the regions in each piece of chewed gum were grouped into a set of four classes according to colour: pink, yellow, mixed and background.

The implemented algorithm was divided into the following steps:

1. 'Read two input images': the first is the front of the chewed wafer (side a) and the second is the opposite side (side b).

2. 'Select a region of interest': create bounding boxes around each wafer and crop the image.

3. 'Convert image from RGB to $\mathrm{L}^{*} \mathrm{a} * \mathrm{~b}$ colour space': $\mathrm{L}^{*}$ represents brightness, ranging from $0-100$, while $\mathrm{a}^{*}$ and $\mathrm{b}^{*}$ represent the colour opponents, green-red and blueyellow. This colour model encompasses the entire spectrum, including colours outside human vision. Only $a^{*}$ and $b^{*}$ layers were used for the analysis to guarantee 


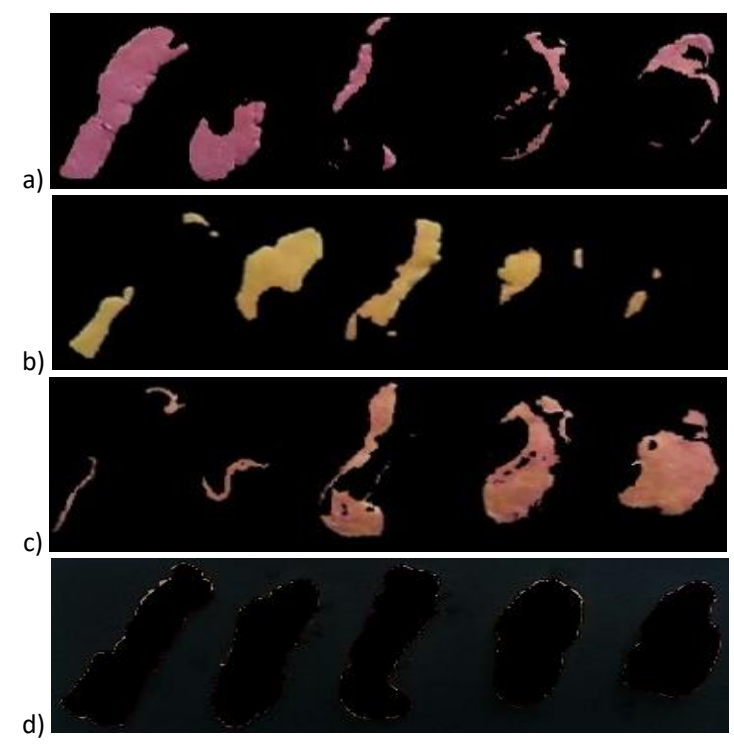

Figure 2. Labelled clusters: a) pink, b) yellow, c) mixed and d) background.

uniform intensity values and preserve the integrity of the colour information.

4. 'Classify the colours in the $a^{*} b^{*}$ space using K-means clustering': this approach assumes each region has a location in space and partitions them into a K-number group of data (clusters) using the Euclidean distance metric. In this way, the objects within each cluster are as close to each other as possible and as far from objects in other clusters as possible (Figure 1-d). In this step, it is necessary to specify the number of $K$ clusters to be partitioned and the number of times clustering using a new initial cluster centroid position should be repeated. In particular, the number of clusters is initially a userspecified parameter according to the number of clusters desired (in this case, four $\mathrm{K}$ clusters to partition the image into the four colour classes, as mentioned previously). Then, each pixel is assigned to a centroid for each class, and this is repeated until the intra-cluster variance is minimised and no points change cluster.

5. 'Pixel labelling': labelling every pixel in the image using the index corresponding to a cluster for every input object (Figure 2).

6. 'Create segmented images': separate objects using pixel labels.
7. 'Calculate area': estimate the area in pixels of the objects in the $\mathrm{K}$ clusters.

8. 'Save segmented images': save and show the images segmented by means of the colours.

A scheme of the implemented algorithm is shown in Figure 3.

\subsection{Subject population}

In our experimental study, six patients were selected and grouped according to their dental status (Table 1):

Group 1:

- Pt. 1: n. teeth $>20$

- Pt. 2: n. teeth $=20$

- Pt. 3: n. teeth $=20$ and RPD - congruent condition

Group 2:

- Pt. 4: n. teeth $<20$ and RPD - congruent condition

- Pt. 5: n. teeth $=20$ and RPD - incongruent condition

- Pt. 6: n. teeth $<20$

In this paper, we considered the first three patients to have a healthy dental status (Group 1) and the other three to have a pathological dental condition (Group 2).

We used the 'two-colour mixing' test, mentioned above, to evaluate to what extent the masticatory performance had been reduced in patients with a minimum number of teeth.

In addition, we assessed whether RPDs allowed any significant improvement in masticatory performance in patients with a minimum number of teeth.

\section{RESULTS}

The images of the samples (on both sides) collected from the subject population according to the described methodology were analysed by means of the K-means clustering system, providing the following results.

\subsection{Clustering results}

By means of the segmentation algorithm, it was possible to estimate the mixed and unmixed areas of the chewing gum. Each colour area was returned as the sum of the partitioned pixels and, for each of the patients, the mixed fraction (MF) and unmixed fraction (UF) of the two colours at 5, 10, 20, 30 and 50 chewing cycles (Figure 3) were calculated and normalised according to the following equations:

$M F=\frac{P i x_{m, a}+P i x_{m, b}}{P i x_{a l l}}$

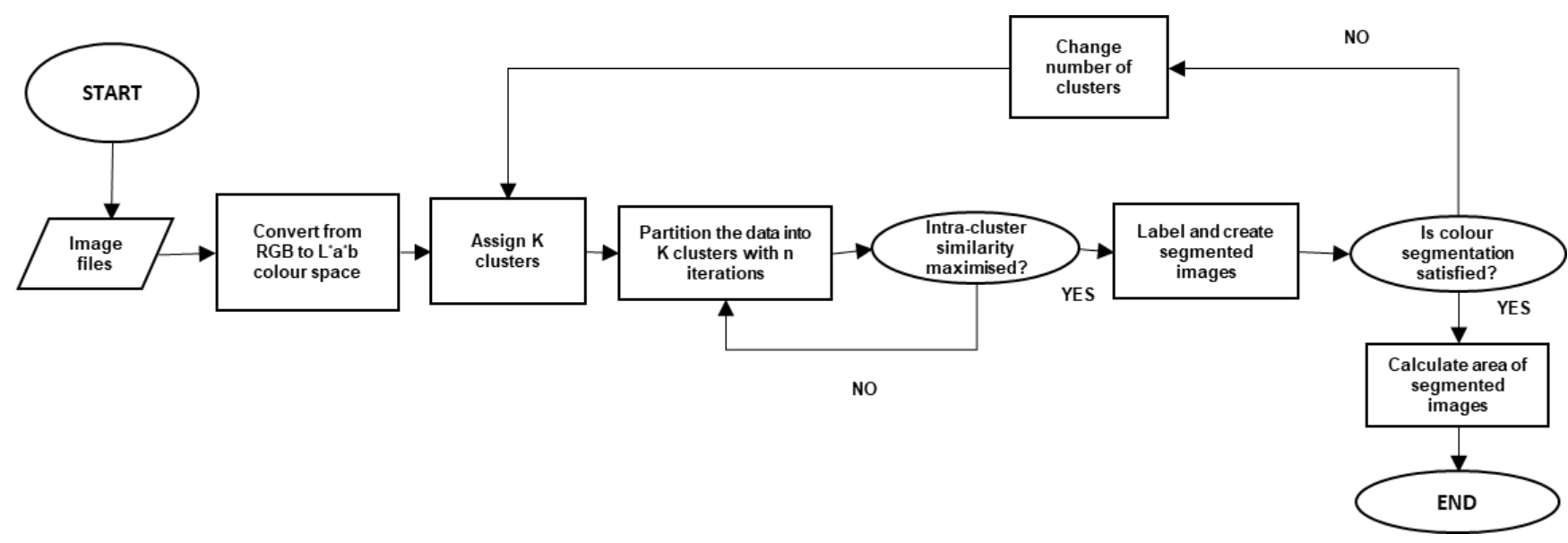

Figure 3. Block diagram of the implemented algorithm. 
Table 1. Overview of styles and font sizes used in this template.

\begin{tabular}{|c|c|c|c|c|c|c|c|c|c|c|c|}
\hline N. teeth & Age & $\begin{array}{l}\text { Chewing } \\
\text { difficulty }\end{array}$ & $\begin{array}{c}\text { Missing } \\
\text { teeth }\end{array}$ & $\begin{array}{c}\text { Decayed } \\
\text { teeth }\end{array}$ & Filled teeth & $\begin{array}{c}\text { Mobile } \\
\text { teeth }\end{array}$ & FPD & RPD & Periodontitis & $\begin{array}{c}\text { Remaining } \\
\text { teeth }\end{array}$ & $\begin{array}{c}\text { Tot. chewing } \\
\text { teeth }\end{array}$ \\
\hline$>20$ & 68 & No & 4 & 0 & 8 & 0 & No & No & No & 28 & 28 \\
\hline$=20$ & 67 & Moderate & 11 & 2 & 2 & 0 & No & No & No & 21 & 21 \\
\hline$=20$ & 85 & Moderate & 11 & 2 & 2 & 2 & Yes & Cong. & Yes & 21 & 26 \\
\hline$<20$ & 84 & No & 19 & 0 & 0 & 0 & Yes & Cong. & No & 13 & 30 \\
\hline$=20$ & 68 & No & 12 & 1 & 4 & 0 & Yes & Incong. & No & 20 & 26 \\
\hline$<20$ & 67 & No & 16 & 0 & 0 & 0 & Yes & No & No & 16 & 16 \\
\hline
\end{tabular}

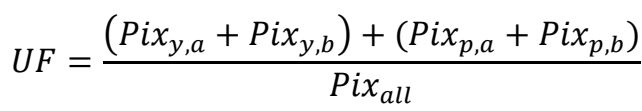

where

- Pix $x_{\mathrm{m}, \mathrm{a}}$ and Pix $\mathrm{m}, \mathrm{b}$ are pixels from each side of the mixed area,

- Pix $/ \mathrm{y}, \mathrm{a}$ and $P i x_{\mathrm{p} / \mathrm{y}, \mathrm{b}}$ are pixels from each side of the pink or yellow area,

- Pix all is the total pixel area.

The means and standard deviations of the MF for all the patients for each chewing cycle (30 samples considered) are summarised in Table 2.

It can be seen that the MF parameter increases with the increase in the number of masticatory cycles.

The table also shows that the MF mean values at 20 chewing cycles indicate a very good agreement with the different dental status of patients: $M F=0.438 \pm 0.214$ (min: 0.126 , $\max : 0.740$ ). This is very important since it is possible to reduce the number of chewing cycles from 50 (typical in other studies [14], [22]-[25]) to 20 to measure $\mathrm{MF}$ parameters that are well correlated to masticatory efficiency. This value was used for the second series of experiments.

In Figure 4, the $M F$ values obtained for each of the patients with different masticatory efficiency are reported as a function of the number of chewing cycles, and the linear regression analysis of the two groups of patients is reported.

The first set of MF values $\left(R^{2}=0.920\right)$, relating to the three participants with a healthy dental status, is more sensitive to the increase in the number of masticatory cycles than the second $\left(\mathrm{R}^{2}\right.$ $=0.91)$, which relates to the three patients with a diseased dental status.

\subsection{Masticatory performance}

The acquired data and their elaboration by means of the $\mathrm{K}$ means method were used to evaluate the differences in masticatory efficiency between the six selected patients. For this purpose, we assessed the masticatory performance (MP) as the UF of each patient. Considering the high variability of the values between the participants at 20 chewing cycles due to the different dental status, for this evaluation we set the fixed number of cycles at $n=20$ (Figure 5).

Table 2. Means and standard deviations of $M F$ for each number of chews.

\begin{tabular}{ccl}
\hline Chewing cycles & MF Means & s.d. \\
\hline 5 & 0.147 & 0.078 \\
10 & 0.313 & 0.126 \\
20 & 0.438 & 0.214 \\
30 & 0.630 & 0.173 \\
50 & 0.769 & 0.169 \\
\hline
\end{tabular}

It is possible to observe how much lower the UF values are compared with the $M P$ values reported for Group 1 patients.

\subsection{Validity}

A comparison between the proposed segmentation algorithm and ViewGum software, a commercial software used for the evaluation of the chewed gum [15], was performed to validate the obtained results. MP, considered as the measure of the unmixed colour and based on the results of both methods for the different chewing cycles for the six patients, is shown in Figure 6. Concerning the segmentation results obtained by ViewGum, the degree of colour mixture was provided as the standard deviation of hue (SDHue) in the HIS cylindrical coordinate system after the selection of the gum surface [15]. In this study, the SDHue represents the UF measured by means of this commercial software. The normalised MP obtained by the UF data processed by the two methods is compared in Figure 6.

It is possible to observe that the proposed method is more sensitive $(+75 \%)$ to masticatory performance than the results obtained by the ViewGum software.

In Figure 7, the UF of the six patients at 20 chewing cycles is calculated and compared with the SDHue value provided by the

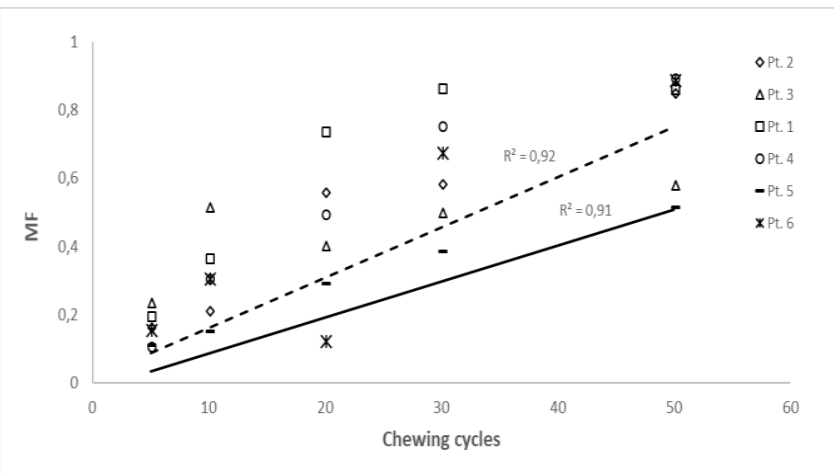

Figure 4. Mixed fraction (MF) for each patient for different chewing cycles and interpolating curves for Group 1 (dotted line) and Group 2 (continuous line).

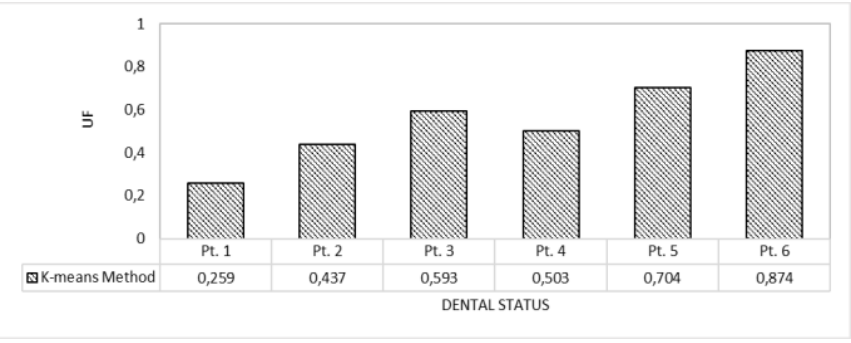

Figure 5. UF calculated by the K-means method at 20 chewing cycles for the six patients. 


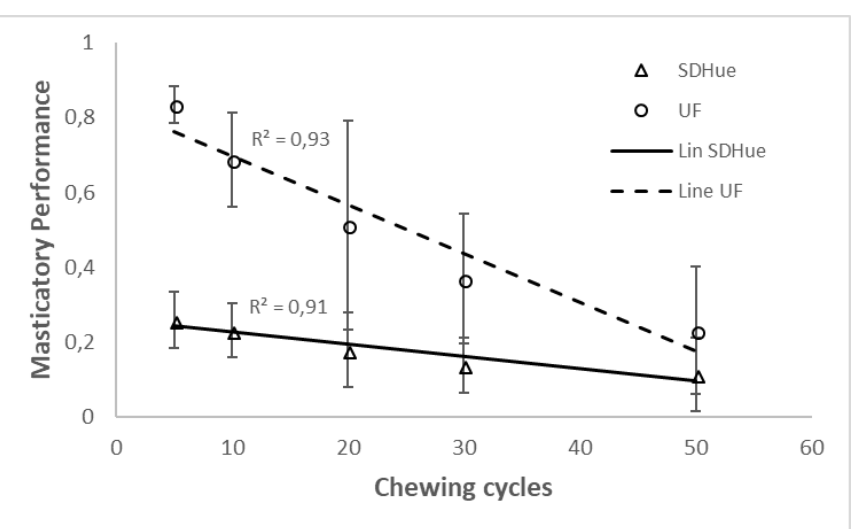

Figure 6. Comparison between the K-means and reference software (ViewGum) data: masticatory efficiency vs chewing cycles.

reference software at the same number of cycles. A higher correlation coefficient $\left(R^{2}=0.89\right)$ is reported for the K-means method than for the data provided by the reference software $\left(R^{2}\right.$ $=0.56$ ).

Finally, in Figure 8, the correlation between the SDHue obtained by the reference software and the UF data obtained by the K-means clustering method for the six selected patients for all the chewing cycles is reported.

It is clear that the two indexes, calculated at 20 chewing cycles, are correlated $\left(R^{2}=0.64\right)$.

\section{DISCUSSION}

\subsection{Test food}

The use of these two gums for the test is appropriate to distinguish the different colour characteristics in the images and determine the MF and UF for each wafer. Furthermore, it has been verified that the proposed algorithm can segment the foreground from the background perfectly using these image colours.

A limitation is the difficulty of cutting a bolus to a thickness of $1 \mathrm{~mm}$ homogeneously to reduce the margin of error during the acquisition phase. In addition, the irregular shape of the wafers indicates the need for a mechanism that allows all samples to be flattened to the same thickness without the loss of information from inadequate flattening.

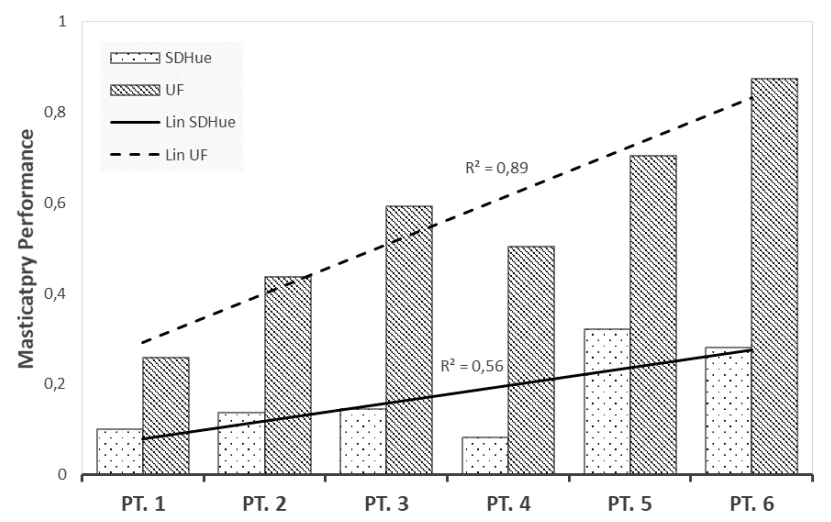

Figure 7. Comparison between the K-means and reference software data at 20 chewing cycles.

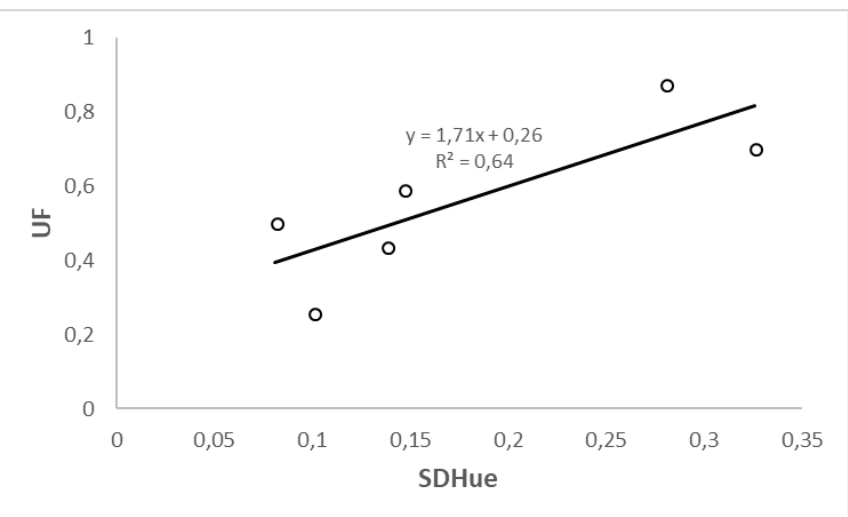

Figure 8. Masticatory performance according to both methods.

\subsection{Segmentation assessment}

Using K-means clustering techniques for the evaluation, the MP for each wafer was evaluated by estimating the mixed and unmixed areas (MF and UF) of the chewing gum. This clustering system, using colour information, provides a high level of discrimination for the regions present in each image, reducing the errors resulting from manual segmentation. It is possible to see a significant increase in colour mixing depending on the number of chewing cycles, as demonstrated in previous studies [12], [15], [16].

The high variability between the MF shown in Figure 4 resulting from different dental status can provide some indication of the MP of each patient. In particular, at 20 chewing cycles, it is possible to see that the MP decreases for the three patients with a diseased dental condition.

\subsection{Comparison with other clinical methods}

In this study, our colour segmentation method was compared with the previously described image segmentation provided by ViewGum software.

The higher discriminating power of the two mixed and unmixed colours was evident. Linear regression analysis demonstrates that the $\mathrm{K}$-means method $\left(R^{2}=0.931\right)$ can provide a better sensitivity compared with ViewGum software results $\left(R^{2}\right.$ $=0.913)$. A Pearson correlation between the two measures was very positive $\left(R^{2}=0.992, p=0.02\right)$. It was observed that the UF varied over a larger range among the participants than SDHue, which lowered the correlation between the two measures.

\subsection{Clinical applicability}

We investigated the capability of this method for carrying out oral health surveys. The six selected patients in our study were recruited for their reduced dentition and their different oral conditions. The large variability in MF for each patient demonstrated that the proposed method provided a good evaluation of chewing function. In particular, the large standard deviations in the results demonstrated the high sensitivity of this image processing technique for different dental status, especially at 20 chewing cycles, which was consequently used as a reference for subsequent investigations.

It is evident that chewing efficiency decreases in patients with 20 teeth, but it remains adequate. The reduction in MF is more pronounced in patients with less than 20 teeth and without RPD, according to Naka [4]. However, the patients did not report masticatory difficulties. This is probably due to the small study sample, but we cannot underestimate the adaptability of the older population; they often believe that their difficulties are problems 
that must be compensated for and are accepted as the normal consequences of aging.

RPDs did not lead to significant improvements in the MF for patients with 20 teeth; indeed, we did not observe any great difference in patients with the same number of natural teeth but without RPD.

We observed an evident difference in chewing efficiency between the patient with 20 teeth and adequate RPD and the patient with the same number of teeth but with incongruous RPD. This is probably due to the discomfort created by an incongruous prosthesis that negatively influences chewing efficiency despite having 20 teeth.

In fact, masticatory function is better when partial dentures are well constructed, but most patients wear resin prostheses with problems in construction, which are badly adapted to the tissues. During mastication, the incongruity of the prosthesis is such as to reduce chewing efficiency to very low levels, but not lower than for patients with less than 20 teeth.

However, prosthetic rehabilitation for patients with less than 20 teeth is fundamental since chewing efficiency improves substantially in the presence of adequate RPD.

\section{CONCLUSION}

In conclusion, this automatic segmentation method has been shown to be capable of quantifying the degree of colour mixing. The advantages of this method are that it is fast, robust and not too complicated to perform. It requires a priori specification of the number of cluster centres, which is often difficult to calculate. In this study, the number was selected by the user, who could change it if the segmentation was not satisfactory.

However, our study has several limitations; the small number of enrolled patients cannot provide statistical information on the different dental conditions, and the results for other chewing gum colours have not been investigated, so these evaluations are valid only for the specific chewing-gum colours mentioned.

However, as a pilot study, the results obtained can be easily combined with data in a larger study for further evaluation.

\section{ACKNOWLEDGEMENT}

The authors would like to thank Prof. Enrico Primo Tomasini for initiating the collaboration and the CARMELO (Center for Advanced Research on Measurements for Engineering and Life Optimization) Research and Development Center for having instigated the research activity.

\section{REFERENCES}

[1] World Health Organization (WHO), World report on ageing and health, 2015, 246 p. Online [Accessed 21 June 2021]

https://apps.who.int/iris/handle/10665/186463?localeattribute $=$ en\&

[2] M. Chen, R. M. Andersen, D. E. Barmes, M. H. Leclerq, C. S, Lyttle, Comparing oral health care systems: a second international collaborative study, WHO, 1997, $350 \mathrm{p}$.

[3] P. E. Petersen, D. Kandelman, S. Arpin, H. Ogawa, Global oral health of older people - call for public health action, Community Dental Health 27 (2010), pp. 257-267. Online [Accessed 21 June 2021] https://pubmed.ncbi.nlm.nih.gov/21313969/

[4] O. Naka, V. Anastassiadou, A. Pissiotis, Association between functional tooth units and chewing ability in older adults: a systematic review, Gerodontology 31 (2014), pp. 166-177. DOI: $\underline{10.1111 / \text { ger.12016 }}$
[5] M. Waas, J. Meeuwissen, R. Meeuwissen, A. Käyser, W. Kalk, M. Hof, Relationship between wearing a removable partial denture and satisfaction in the elderly, Community Dentistry and Oral Epidemiology 22 (1994), pp. 315-318.

DOI: $10.1111 / j .1600-0528.1994 . t b 02059 . x$

[6] A. Sheiham, J. G. Steele, W. Marcenes, C. Lowe, S. Finch, C. J. Bates, A. W. G. Walls, The relationship among dental status, nutrient intake, and nutritional status in older people, Journal of Dental Research 80 (2001), pp. 408-413. DOI: $10.1177 / 00220345010800020201$

[7] G. Boretti, M. Bickel, A. H. Geering, A review of masticatory ability and efficiency, Journal of Prosthetic Dentistry 74 (1995), pp. 400-403.

DOI: $10.1177 / 00220345010800020201$

[8] A. A. Bajoria, S. Saldanha, V. K. Shenoy, Evaluation of satisfaction with masticatory efficiency of new conventional complete dentures in edentulous patients - a survey, Gerodontology 29 (2012), pp. 231-238. DOI: $10.1111 / \mathrm{j} .1741-2358.2012 .00634 . \mathrm{x}$

[9] L. Eberhard, H. J. Schindler, D. Hellmann, M. Schmitter, P. Rammelsberg, N. N. Giannakopoulos, Comparison of particlesize distributions determined by optical scanning and by sieving in the assessment of masticatory performance, Journal of Oral Rehabilitation 39 (2012), pp. 338-348.

DOI: $10.1111 / \mathrm{j} .1365-2842.2011 .02275 . \mathrm{x}$

[10] M. Bessadet, E. Nicolas, M. Sochat, M. Hennequin, J. L. Veyrune, Impact of removable partial denture prosthesis on chewing efficiency, Journal of Applied Oral Science 21 (2013), pp. 392-396. DOI: $10.1590 / 1679-775720130046$

[11] S. Sato, K. Fueki, H. Sato, S. Sueda, T. Shiozaki, M. Kato, T. Ohyama, Validity and reliability of a newly developed method for evaluating masticatory function using discriminant analysis, Journal of Oral Rehabilitation 30 (2003), pp. 146-151. DOI: $\underline{10.1046 / \mathrm{j} .1365-2842.2003 .01050 . \mathrm{x}}$

[12] M. S. Kaya, B. Güçlü, M. Schimmel, S. Akyuz, Two-colour chewing gum mixing ability test for evaluating masticatory performance in children with mixed dentition: validity and reliability study, Journal of Oral Rehabilitation 44(11) (2017), pp. 827-834.

DOI: $10.1111 /$ joor. 12548

[13] B. Liedberg, O. Ekberg, B. Öwall, Chewing and the dimension of the pharyngoesophageal segment, Dysphagia 6 (1991), pp. 214218. DOI: $10.1007 / \mathrm{BF} 02493530$

[14] J. F. Prinz, Quantitative evaluation of the effect of bolus size and number of chewing strokes on the intra-oral mixing of a twocolour chewing gum, Journal of Oral Rehabilitation 26 (1999), pp. 243-247.

DOI: $10.1046 / j .1365-2842.1999 .00362 . x$

[15] D. J. Halazonetis, M. Schimmel, G. S. Antonarakis, P. Christou, Novel software for quantitative evaluation and graphical representation of masticatory efficiency, Journal of Oral Rehabilitation 40 (2013), pp. 329-335.

DOI: $10.1111 /$ joor:12043

[16] M. Schimmel, P. Christou, H. Miyazaki, D. Halazonetis, F. R. Herrmann, F. Müller, A novel colourimetric technique to assess chewing function using two-coloured specimens: validation and application, Journal of Dentistry 43 (2015), pp. 955-964. DOI: $\underline{10.1016 / \text { i.jent.2015.06.003 }}$

[17] D. Mery, F. Pedreschi, Segmentation of colour food images using a robust algorithm, Journal of Food Engineering 66 (2005), pp. 353-360.

DOI: $10.1016 / j . j$ foodeng.2004.04.001

[18] M. Steinbach, V. Kumar, P. Tan, Cluster analysis: basic concepts and algorithms, in: Introduction to Data Mining. Pearson Addison Wesley, Boston, 2005, ISBN-13: 9780133128901, pp. 125-146

[19] N. Dhanachandra, K. Manglem, Y. J. Chanu, Image segmentation using K-means clustering algorithm and subtractive clustering algorithm, Procedia Computer Science 54 (2015), pp. 764-771. DOI: $10.1016 /$ i.procs.2015.06.090 
[20] J. MacQueen, Some methods for classification and analysis of multivariate observations, Proc. of the $5^{\text {th }}$ Berkeley Symposium on Mathematical Statistics and Probability, Berkeley, USA, 21 June 18 July 1965 , pp. 281-297.

[21] E. Holvoet, K. Vanden Wyngaert, A. H. Van Craenenbroeck, W. Van Biesen, S. Eloot, The screening score of Mini Nutritional Assessment (MNA) is a useful routine screening tool for malnutrition risk in patients on maintenance dialysis, PLoS ONE 15 (2020), e0229722. DOI: 10.1371 /journal.pone.0229722

[22] V. Anastassiadou, M. R. Heath, The development of a simple objective test of mastication suitable for older people, using chewing gums, Gerodontology 18 (2001), pp. 79-86.
DOI: $10.1111 / \mathrm{j} .1741-2358.2001 .00079 . \mathrm{x}$

[23] R. Manly, S. L. Braley, Masticatory performance and efficiency, Journal of Dental Research 29 (1950), pp. 448-462. DOI: $10.1177 / 00220345500290040701$

[24] I. Hayakawa, I. Watanabe, H. Shigezo, N. Masanori, S. Tetsuya, A simple method for evaluating masticatory performance using a colour-changeable chewing gum, International Journal of Prosthodontics 11 (1998), pp. 173-176.

[25] K. Hirano, S. Hirano, H. Iwao, The role of oral sensorimotor function in masticatory ability, Journal of Oral Rehabilitation 31 (2004), pp. 199-205.

DOI: $10.1111 / \mathrm{i} .1365-2842.2004 .01175 . \mathrm{x}$ 